\title{
An alarming rise of non-albicans Candida species and uncommon yeasts in the clinical samples; a combination of various molecular techniques for identification of etiologic agents
}

\author{
Monireh Taei ${ }^{1}$, Mostafa Chadeganipour ${ }^{1}$ and Rasoul Mohammadi ${ }^{12^{*}}$ (1)
}

\begin{abstract}
Objective: Yeasts are unicellular microorganisms may cause systemic infection in immunocompromised patients. The aim of this study was to identify yeast strains isolated from clinical specimens using molecular techniques.

Results: A total of 202 yeast strains isolated from 341 clinical samples between February 2017 and May 2019. All clinical isolates were identified using phenotypic and molecular tests including PCR-RFLP, duplex-PCR, multiplex-PCR, and PCR-sequencing. The most yeast fungal isolates were obtained from urine (66.8\%), nail (9.4\%), skin lesion (7.9\%), bronchoalveolar lavage (5.9\%), and blood (3.9\%). One hundred and twenty-one Candida species were identified as non-albicans versus 76 Candida albicans. Trichosporon asahii, and Pichia terricola were uncommon non-Candida yeasts isolated from urine samples. For the first time, we isolated $P$. terricola as etiological agent of urinary tract infection in a pregnant female. Since Candida species show different levels of resistance to antifungal agents, precise identification of clinical isolates is critical for better treatment of infection.
\end{abstract}

Keywords: Non-albicans Candida species, Uncommon yeasts, Trichosporon asahii, Pichia terricola, Identification, Molecular techniques

\section{Introduction}

Candida species are the most common cause of fungal infections worldwide. They are the third most dominant cause of healthcare-related infections [1]. Candida albicans is the most common species in humans; however, the increasing of non-albicans Candida (NAC) species have been recognized significantly during the last two decades [2-4]. The most NAC infections are caused by C. glabrata, C. tropicalis, C. parapsilosis, C. dubliniensis, C. guilliermondii, C. krusei, and C. kefyr [5-7]. The growing number of NAC species might be connected to former exposure to polyenes and azoles, use of indwelling catheters, malignancies, age, the improved biochemical

\footnotetext{
*Correspondence: Dr.rasoul_mohammadi@yahoo.com

1 Department of Medical Parasitology and Mycology, School of Medicine, Isfahan University of Medical Sciences, Isfahan, Iran

Full list of author information is available at the end of the article
}

and molecular diagnostic methods in laboratories, and geographical regions [8, 9]. As compared with C. albicans, this epidemiological pattern towards NAC species with high MICs of azoles and triazoles, appears among high-risk patients due to the common and prolonged use of antifungal agents [10]. High mortality rate (30-50\%), along with raising resistance to antifungal agents among NAC species, imposes serious medical and economic problems to the society $[7,11]$. The raising frequency of NAC species has been repeatedly reported from different areas worldwide, for example, C. tropicalis is frequently isolated in Asia and South America and C. glabrata has a high frequency in North and Central Europe and United States of America specially, among the elderly people [12]. Due to the increase reports of uncommon yeast infection and their antifungal resistance by physicians in our region, the present study was undertaken to investigate the frequency of various Candida species and rare 
yeasts collected from fungal infections in Kashani university hospital and Shefa Lab (referral medical mycology laboratory), in Isfahan, Iran, by combination of miscellaneous molecular techniques.

\section{Main text \\ Materials and methods \\ Patients and sampling}

A total of 341 suspected cases ( 75 male and 266 female) referred to the Kashani university hospital and a referral medical mycology laboratory (Shefa Lab.), in Isfahan, Iran, from February 2017 to May 2019. Demographic data were documented for each subject. Excluding criteria were considered for the patients who had taken antifungal agents during the last week.

\section{Primary screening}

All specimens were examined by direct microscopy with $10 \%$ Potassium Hydroxide ( $\mathrm{KOH} 10 \%$ ), sub-culture on sabouraud dextrose agar (Biolife, Italy), and CHROMagar Candida (France).

\section{Molecular methods as secondary screening for identification of Candida species Polymerase chain reaction-restriction fragment length polymorphism (PCR-RFLP) for identification of six prevalent species of Candida}

This screening was done in an iterative manner (Additional file 1: Figures S1-S6).

Genomic DNA of isolates was extracted and ITS15.8SrDNA-ITS2 region was amplified. Briefly, the ITS15.8SrDNA-ITS2 region was amplified by a PCR mixture including $5 \mu \mathrm{L}$ of $10 \times$ reaction buffer, $0.4 \mathrm{mM}$ dNTPs, $1.5 \mathrm{mM} \mathrm{MgCl} 2,2.5 \mathrm{U}$ of Taq polymerase, 30 pmol of both ITS1 (5'-TCC GTA GGT GAA CCT GCG G-3') and ITS4 (5'-TCC TCC GCT TAT TGA TAT GC-3') primers and $2 \mu \mathrm{L}$ DNA in a final volume of $50 \mu \mathrm{L}$. The PCR cycling conditions were: an initial denaturation phase at $94{ }^{\circ} \mathrm{C}$ for $5 \mathrm{~min}$, followed by 32 cycles of denaturation at $94{ }^{\circ} \mathrm{C}$ for $30 \mathrm{~s}$, annealing at $55{ }^{\circ} \mathrm{C}$ for $45 \mathrm{~s}$, and extension at $72{ }^{\circ} \mathrm{C}$ for $1 \mathrm{~min}$, with a final extension phase at $72^{\circ} \mathrm{C}$ for $7 \mathrm{~min}$. PCR products were digested with the restriction enzyme MspI (Fermentas, Vilnius, Lithuania). Five microliter of each PCR amplicons and $10 \mu \mathrm{L}$ of RFLP products were separated by gel electrophoresis on $1.5 \%$ and $2 \%$ agarose gel (containing $0.5 \mu \mathrm{g} / \mathrm{mL}$ ethidium bromide), respectively. In this stage, all clinical isolates first grouped into 6 prevalent species as Candida albicans, C. tropicalis, $C$. parapsilosis complex, C. glabrata, C. krusei, and C. guilliermondii [13].
Duplex-PCR assay for distinction of C. albicans and C. dubliniensis

Species-specificity primers were used to identify $C$. albicans and $C$. dubliniensis. The sequences of these primers are as follows:

CALF (5'-TGGTAAGGCGGGATCGCTT-3') and CALR (5'-GGTCAAAGTTTGAAGATATAC) for $C$. albicans, and CDUF (5'-AAACTTGTCACGAGATTA TTTTT) and CDUR (5'-AAAGTTTGAAGAATAAAA TGGC-3') for C. dubliniensis. The size of PCR products for C. albicans and C. dubliniensis are $100 \mathrm{bp}$, and $325 \mathrm{bp}$, respectively [14].

\section{Multiplex-PCR for differentiation of Candida glabrata and its phylogenetically related species Candida bracarensis and Candida nivariensis}

Targeting the ITS1 region, reverse primer UNI-5.8S $\left(5^{\prime}\right.$ ACCAGAGGGCGCAATGTG $3^{\prime}$ ) and forward primers GLA-f (5' CGGTTGGTGGGTGTTCTGC $\left.3^{\prime}\right)$, NIV-f ( $5^{\prime}$ AGGGAGGAGTTTGTATCTTTCAAC $3^{\prime}$ ), and BRA-f (5' GGGACGGTAAGTCTCCCG 3'), were applied for identification of C. glabrata, C. nivariensis, and C. bracarensis, respectively. Expected amplicon size for $C$. glabrata, C. nivariensis, and C. bracarensis are $379 \mathrm{bp}$, $293 \mathrm{bp}$, and $223 \mathrm{bp}$, respectively [15].

\section{Multiplex-PCR for differentiation of Candida parapsilosis complex}

Three pairs of primers including CPAF $\left(5^{\prime}\right.$ TTTGCT TTGGTAGGCCTTCTA $3^{\prime}$ ) and CPAR (5' GAGGTC GAATTTGGAAGAAGT $3^{\prime}$ ), CORF (5' TTTGGTGGC CCACGGCCT $\left.3^{\prime}\right)$ and CORR ( $5^{\prime}$ TGAGGTCGAATT TGGAAGAATT $3^{\prime}$ ), and CMEF (5' TTTGGTGGG CCCACGGCT $3^{\prime}$ ) and CMER (5' GAGGTCGAATTT GGAAGAATGT $3^{\prime}$ ), were used for identification of Candida parapsilosis, C. orthopsilosis, and C. methapsilosis, respectively. The size of amplicons for Candida parapsilosis, C. orthopsilosis, and C. methapsilosis are $379 \mathrm{bp}, 367 \mathrm{bp}$, and $374 \mathrm{bp}$, respectively [16].

\section{PCR-sequencing}

All PCR products with no cutting site for the restriction enzyme MspI considered as non-Candida yeasts, and were applied for sequence analysis. The non-Candida yeasts amplicons were purified using the ethanol purification method, and cycle sequencing reactions in forward direction were performed (Bioneer, South Korea). The sequencing products were analyzed with Chromas 2.3 (http://chromas.software.informer.com/2.4/), and were evaluated by using of NCBI BLAST searches 
against fungal sequences existing in DNA databases (http://blast.ncbi.nlm.nih.gov/Blast.cgi).

\section{Data analysis}

The results of phenotypic and genotypic tests were analyzed by Chi square and Fisher's exact test in the SPSS version 23.

\section{Results}

Two hundred and two patients out of 341 suspected cases had yeast fungal infections (59.2\%). Male to female sex ratio was 74/128. Age range of patients was $1-87$ years with the median age range of 42.3. Diabetes (18.8\%), pregnancy (18.3\%), kidney stones (2.9\%), burn (2.9\%), and neoplasm $(2.4 \%)$ were the most leading predisposing factors among patients. The most yeast fungal isolates were obtained from urine $(66.8 \%)$, nail $(9.4 \%)$, skin lesion (7.9\%), bronchoalveolar lavage (5.9\%), and blood (3.9\%) (Fig. 1). The majority of patients hospitalized in ICU (9.9\%), surgical ward (9.9\%), neurological intensive care unit (NICU) (8.9\%), infectious diseases ward (8.9\%), and gynaecology ward (7.4\%). Ninety-six cases (46.5\%) were outpatients. Interestingly, 121 Candida species were identified as non-albicans versus 76 Candida albicans by molecular techniques (Fig. 2). Among NAC species, 92 Candida glabrata (76\%), 8 Pichia kudriavzevii (teleomorph of the Candida krusei) (6.6\%), 7 C. kefyr (5.7\%), 6 C. parapsilosis (4.9\%), 3 C. tropicalis (2.4\%), 1
C. dubliniensis (0.8\%), 1 C. guilliermondii (0.8\%), 1 C. famata (Debaryomyces hansenii) (0.8\%), 1 Kluyveromyces marxianus (teleomorph of the Candida parapsilosis) $(0.8 \%)$, and 1 C. orthopsilosis $(0.8 \%)$ were isolated from clinical samples. Fourteen isolates had no cutting site for the restriction enzyme $M s p \mathrm{I}$ and their amplicon sizes were under $500 \mathrm{bp}$. These clinical isolates were applied for cycle sequencing reactions in forward direction. Table 1 shows the frequency of Candida species in the present study among various clinical specimens in different wards of the hospital.

Fisher's exact test revealed that the associations between the kind of clinical samples and yeasts were not statistically significant $(p=0.85)$.

\section{Discussion}

Although C. albicans is the principal causative agent of candidiasis, the raising of NAC species resistant to antifungal drugs such as echinocandins and fluconazole in patients with nosocomial infections is concerning. Resistance to azoles is infrequent in C. albicans $(<5 \%)$, however, it is more current in C. parapsilosis (4-10\%), C. tropicalis (4-9\%), and C. glabrata (4-16\%) [17]. Increasing of NAC species is due to the improved laboratory diagnosis, previous exposure to polyene and azole agents, use of indwelling medical devices, malignancies, growing number of immunocompromised patients, and long-term immunosuppressive and cytotoxic therapy in

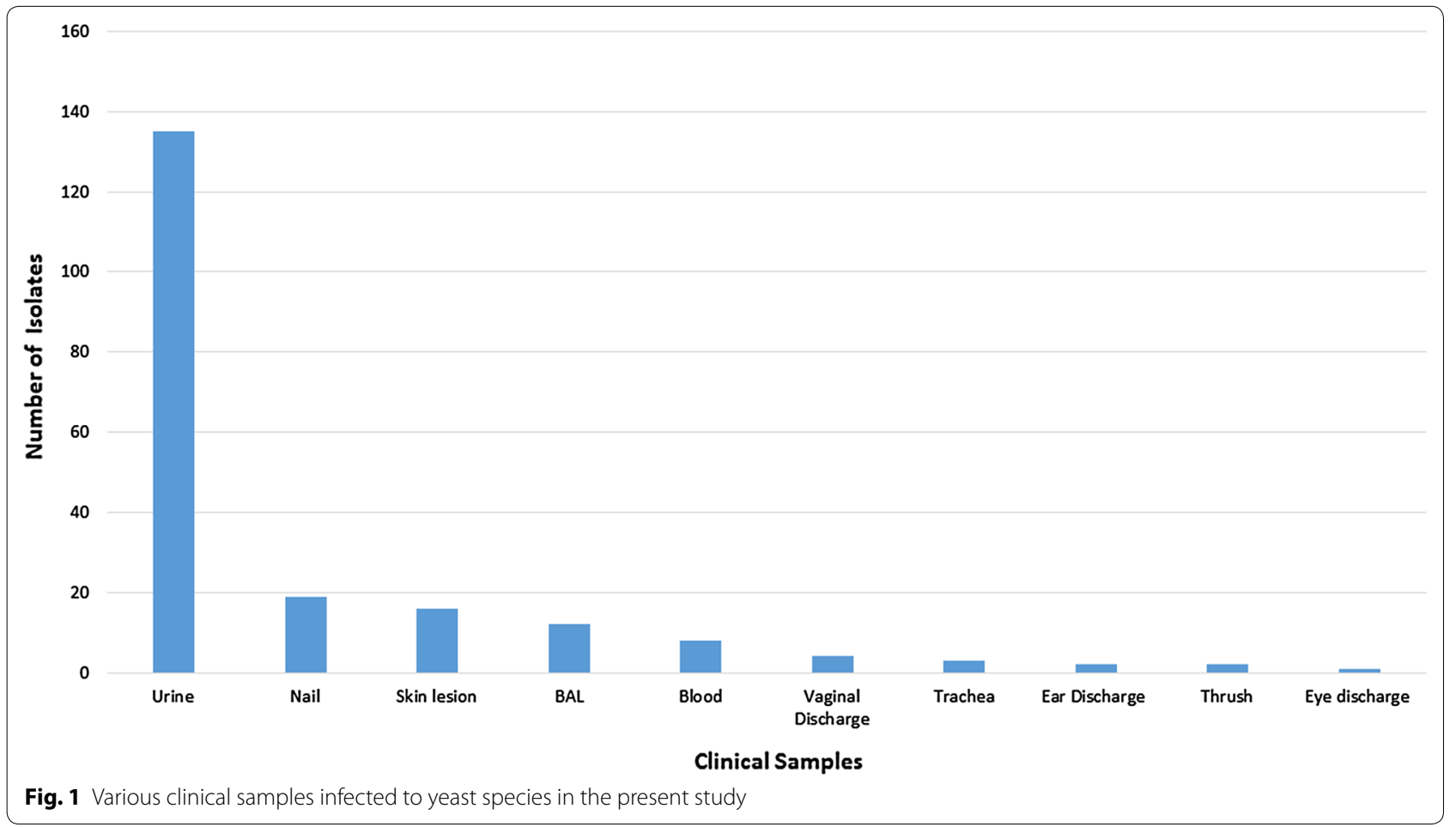



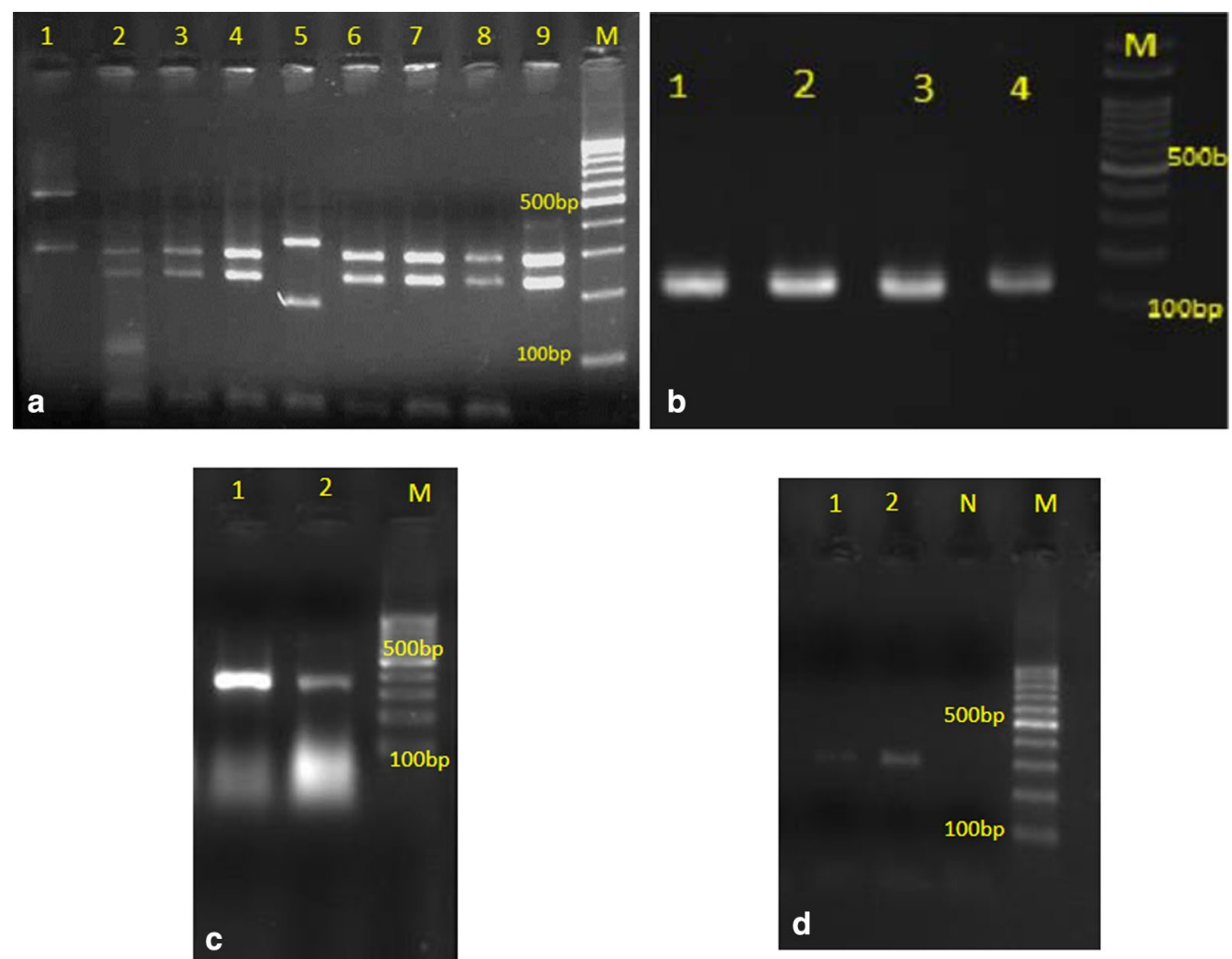

Fig. 2 a Agarose gel electrophoresis of PCR-RFLP products of Candida isolates: lane 1 is C. glabrata, lanes 2-4, and 6-9 are C. albicans, and lane 5 is C. tropicalis; b Agarose gel electrophoresis of duplex-PCR for distinction of C. albicans and C. dubliniensis; lanes 1-4 are C. albicans; $\mathbf{c}$ multiplex-PCR for differentiation of C. parapsilosis complex; lanes 1, 2 are C. parapsilosis; $\mathbf{d}$ multiplex-PCR for differentiation of C. glabrata complex; lanes 1, 2 are C. glabrata; $\mathrm{N}$ is negative control; and $\mathrm{M}$ is $100 \mathrm{bp}$ DNA size marker

organ transplant recipients and cancer patients $[9,18]$. It is usually believed that increasing use of azole treatments in clinics may be associated with the rise of NAC species [19]. The most patients infected to NAC, received empirical antifungals, whereas more $C$. albicans infections were treated when the diagnosis was confirmed. These findings may suggest that NAC infections are in worse clinical conditions [20]. In the last two decades, C. albicans was responsible for over $80 \%$ of etiological agents of all types of candidiasis [21], however, in the present study C. albicans isolated from almost $38.5 \%$ of Candida infections. Candida glabrata is obtained from 15 to $20 \%$ of Candida infections [22,23], but we isolated 92 C. glabrata strains (46.7\%) in the present investigation. In consistent with Singh et al. [24] we revealed a high prevalence of NAC species (61.4\%), but C. albicans was the most repeatedly isolate $(38.5 \%)$. The predominance of C. glabrata among NAC in the present investigation, is contrary to the prospective report by Chakrabarti et al. [25] where C. tropicalis was the most prevalent NAC species (42.1\%). Furthermore, Bhattacharjee reported $C$. tropicalis as the most common NAC in a tertiary care hospital in Kolkata, India [26]. Kaur et al. and Al-Attas and Amro, reported a lower rate of C. glabrata, as well. They showed frequencies of $(10 \%)$ and $(11.1 \%)$ for $C$. glabrata, respectively [27, 28]. Interestingly, Pakshir et al. [29] showed C. parapsilosis as the most prevalent Candida species collected from fingernail infections (45.3\%), even higher than C. albicans (23.7\%). Non-albicans Candida infections may have more incidence among patients with allogeneic hepatocyte transplantation, neutropenia, hematologic malignancies, and severe immune suppression $[30,31]$ however, in our study, there was no difference between neutropenia and malignancies with $C$. albicans or NAC infections. Montagna et al. [32] found that $C$. albicans was more prevalent than NAC species in surgical wards, nevertheless, it was mainly isolated from gynaecology $(n=10)$, ICU $(n=9)$, and infectious diseases $(n=8)$ wards in the present study. According to PCR-RFLP using the restriction enzyme MspI, the differentiation between C. albicans and C. dubliniensis in $C$. albicans complex, C. parapsilosis, C. orthopsilosis, and C. methapsilosis in C. parapsilosis complex, and C. glabrata, C. bracarensis and C. nivariensis in C. glabrata complex 


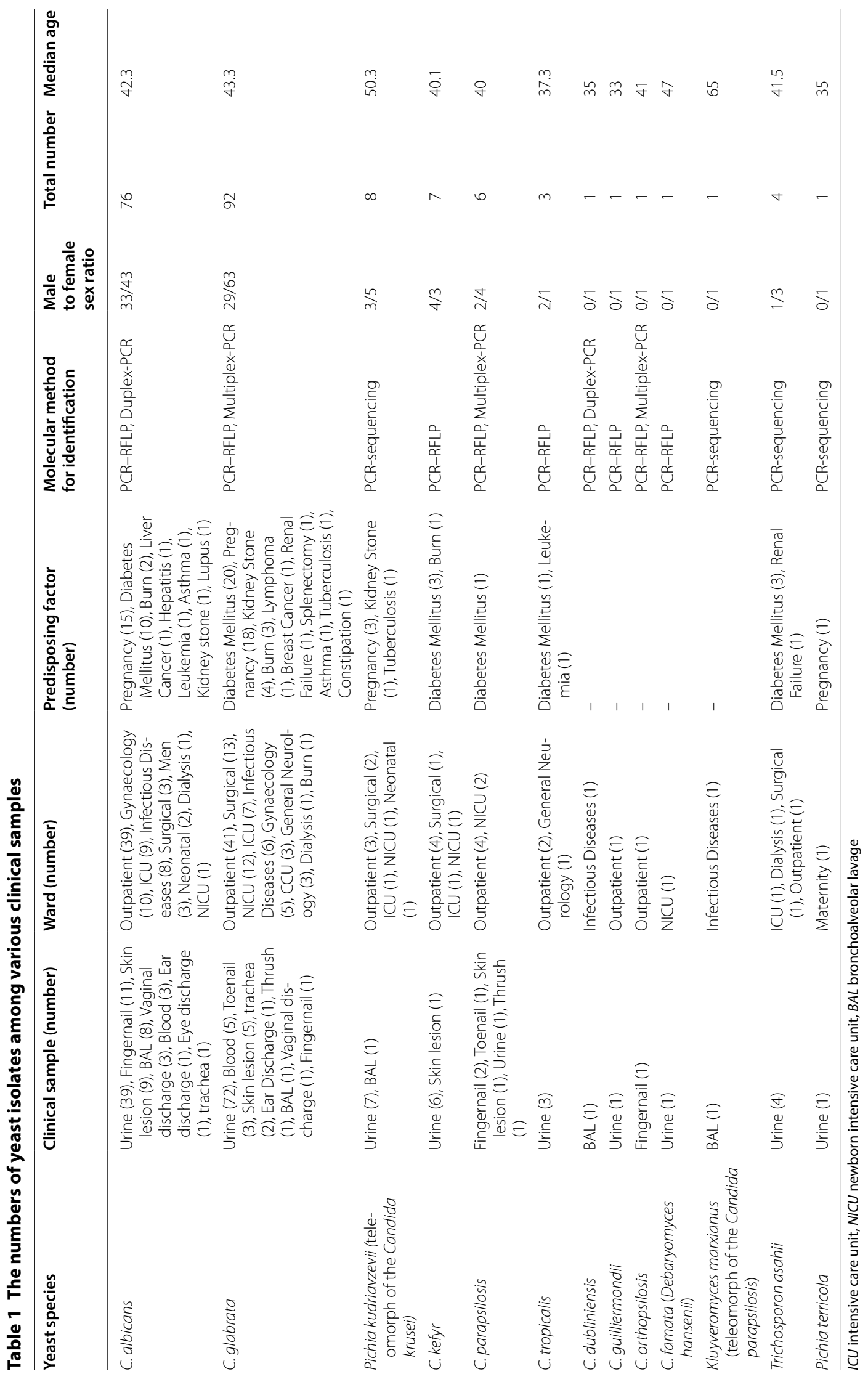


is not possible, so we used effective molecular tests (duplex and multiplex-PCR) for distinguishing them. All strains in C. glabrata complex were C. glabrata, however, one $C$. dubliniensis and one C. orthopsilosis were isolated from C. albicans complex and C. parapsilosis complex, respectively. Candida orthopsilosis is a rare Candida species that reported by Tavanti et al. [33] for the first time. We also identified this species for the first time in Iran in our previous study [34]. In agreement with our previous report, here it was isolated from a patient with fingernail infection. Trichosporon asahii (Trichosporon beigelii) was another uncommon yeast isolated in the present study and identified by PCR-sequencing. This species is connected with a wide spectrum of clinical signs, ranging from superficial lesions in immunocompetent individuals to severe systemic and fatal infections in immunocompromised patients [35]. It is the most prevalent nonCandida cause of fungemia [36], however, all T. asahii in the present study were isolated from urine samples. We also isolated Pichia terricola from clinical sample for the first time. Pichia genus is found in plants, fruit juices, and soil. It has also been described as a normal flora of throat, skin, and alimentary tract. Some species such as $P$. anomala can cause serious infections among immunosuppressed patients mainly in infants [37], however, we isolated $P$. terricola from the urine sample of a pregnant female with severe urinary tract infection.

In conclusion, the contribution of $C$. albicans and NAC species in Candida infections is changing from the past in different areas, and epidemiological data show the increasing of NAC infections worldwide. Due to the increasing of antifungal resistance of Candida species and the elevating number of immuno suppressed patients, it is essential to provide new effective strategies for treatment of this fungal infection. As NAC species have various virulence factors and antifungal susceptibility profile, precise molecular identification can help us to reach to these advantageous strategies.

\section{Limitations}

Due to the sanctions, the most antifungal agents were not found in the country, and if they were, they would be $4-5$ times of the price. We recommend the antifungal susceptibility testing of clinical isolates in further studies.

\section{Supplementary information}

Supplementary information accompanies this paper at https://doi. org/10.1186/s13104-019-4811-1.

Additional file 1. Agarose gel electrophoresis photos of PCR-RFLP taken from primary screening and repeat experiments. Fig S1. Agarose gel electrophoresis for PCR-RFLP; from left to right: C. albicans, C. glabrata, C. albicans, C. glabrata, C. glabrata, C. albicans, C. glabrata, C. albicans, C. glabrata, and 100 bp DNA size Marker. Fig S2. Agarose gel electrophoresis for PCR-RFLP; from left to right: C. glabrata, C. parapsilosis, C. tropicalis, C. albicans, C. albicans, No band, C. albicans, C. albicans (dim band), and 100 bp DNA size Marker. Fig S3. Agarose gel electrophoresis for PCRRFLP; from left to right: C. albicans, C. tropicalis, C. albicans, C. albicans, C. albicans, Mixed bands (C. albicans, and C. kefyr), C. albicans, C. albicans, C. albicans, C. albicans, C. albicans, C. glabrata, C. glabrata, C. tropicalis, C. albicans, and 100 bp DNA size Marker. Fig S4. Agarose gel electrophoresis for PCR-RFLP; from left to right: C. parapsilosis, C. parapsilosis, C. parapsilosis, C. parapsilosis, C. parapsilosis, No band, and 100 bp DNA size Marker. Fig S5. Agarose gel electrophoresis for PCR-RFLP; from left to right: C. albicans, C. albicans, C. albicans, C. albicans, C. albicans, C. albicans, C. albicans, C. albicans, C. albicans, and 100 bp DNA size Marker. Fig S6. Agarose gel electrophoresis for PCR-RFLP; from left to right: C. tropicalis, C. parapsilosis, C. albicans, albicans, albicans, and 100 bp DNA size Marker.

\section{Abbreviations}

NAC: non-albicans Candida; MIC: minimum inhibitory concentration; PCR: polymerase chain reaction; RFLP: restriction fragment length polymorphism.

\section{Acknowledgements}

All authors appreciate Kashani university hospital and Shefa laboratory personnel for their cooperation.

\section{Authors' contributions}

RM design of study; MT, RM, and MC sample collection; RM and MT perform practical tests; RM, and MC data evaluation; and RM preparation of the manuscript. All authors read and approved the final manuscript.

\section{Funding}

This study was supported by Isfahan University of Medical Sciences, Isfahan, Iran, under the Thesis Number 397112. The role of the funding body was in the design of the investigation.

Availability of data and materials

All data generated or analyzed during this study are included in this article.

\section{Ethics approval and consent to participate}

This investigation was approved by the Ethics Committee of Isfahan University of Medical Sciences. The code number is IR.MUI.MED.REC.1397.066.

\section{Consent to publish}

Not applicable.

\section{Competing interests}

The authors declare that they have no competing interests.

\section{Author details}

${ }^{1}$ Department of Medical Parasitology and Mycology, School of Medicine, Isfahan University of Medical Sciences, Isfahan, Iran. ${ }^{2}$ Infectious Diseases and Tropical Medicine Research Center, Isfahan University of Medical Sciences, Isfahan, Iran.

Received: 30 September 2019 Accepted: 14 November 2019

Published online: 29 November 2019

\section{References}

1. Cortegiani A, Misseri G, Chowdhary A. What's new on emerging resistant Candida species. Intensive Care Med. 2019;45(4):512-5.

2. Manfredi M, McCullough M, Al-Karaawi Z, Hurel S, Porter S. The isolation, identification and molecular analysis of Candida spp. isolated from the oral cavities of patients with diabetes mellitus. Oral Microbiol Immunol. 2002:17(3):181-5.

3. Badiee P, Alborzi A, Shakiba E, Ziyaeyan M, Rasuli M. Molecular identification and in vitro susceptibility of Candida albicans and C. dubliniensis isolated from immunocompromised patients. Iran Red Crescent Med J. 2009;11(4):391-7. 
4. Ahmad S, Khan Z, Mokaddas E, Khan ZU. Isolation and molecular identification of Candida dubliniensis from non-human immunodeficiency virus-infected patients in Kuwait. J Med Microbiol. 2004;53(Pt 7):633-7.

5. Pfaller MA, Castanheira M, Messer SA, Jones RN. In vitro antifungal susceptibilities of isolates of Candida spp. and Aspergillus spp. from China to nine systemically active antifungal agents: data from the SENTRY antifungal surveillance program, 2010 through 2012. Mycoses. 2015;58(4):209-14.

6. Pfaller M, Rhomberg P, Messer S, Jones R, Castanheira M. Isavuconazole, micafungin, and 8 comparator antifungal agents' susceptibility profiles for common and uncommon opportunistic fungi collected in 2013: temporal analysis of antifungal drug resistance using CLSI species-specific clinical breakpoints and proposed epidemiological cutoff values. Diagn Microbiol Infect Dis. 2015;82(4):303-13.

7. Kołaczkowska A, Kołaczkowski M. Drug resistance mechanisms and their regulation in non-albicans Candida species. J Antimicrob Chemother. 2016;71(6):1438-50.

8. Pfaller MA, Castanheira M, Messer SA, Moet GJ, Jones RN. Variation in Candida spp. distribution and antifungal resistance rates among bloodstream infection isolates by patient age: report from the SENTRY Antimicrobial Surveillance Program (2008-2009). Diagn Microbiol Infect Dis. 2010;68(3):278-83.

9. Miceli MH, Díaz JA, Lee SA. Emerging opportunistic yeast infections. Lancet Infect Dis. 2011;11(2):142-51.

10. Falagas ME, Roussos N, Vardakas KZ. Relative frequency of albicans and the various non-albicans Candida spp. among candidemia isolates from inpatients in various parts of the world: a systematic review. Int J Infect Dis. 2010;14(11):e954-66.

11. Pereira CA, Toledo BC, Santos CT, Costa ACBP, Back-Brito GN, Kaminagakura E, et al. Opportunistic microorganisms in individuals with lesions of denture stomatitis. Diagn Microbiol Infect Dis. 2013;76(4):419-24

12. Blot S, Vandewoude K, Hoste E, Poelaert J, Colardyn F. Outcome in critically ill patients with candidal fungaemia: Candida albicans vs. Candida glabrata. J Hosp Infect. 2001;47(4):308-13.

13. Mirhendi $H$, Makimura $K$, Khoramizadeh M, Yamaguchi $H$. A one-enzyme PCR-RFLP assay for identification of six medically important Candida species. Nippon Ishinkin Gakkai Zasshi. 2006:47(3):225-9.

14. Ahmad S, Khan Z, Asadzadeh M, Theyyathel A, Chandy R. Performance comparison of phenotypic and molecular methods for detection and differentiation of Candida albicans and Candida dubliniensis. BMC Infect Dis. 2012;25(12):230.

15. Romeo O, Scordino F, Pernice I, Passo CL, Criseo G. A multiplex PCR protocol for rapid identification of Candida glabrata and its phylogenetically related species Candida nivariensis and Candida bracarensis. J Microbiol Methods. 2009;79(1):117-20.

16. Rodríguez $\mathrm{L}$, Jewtuchowicz V. Molecular characterization of Candida parapsilosis species complex in niches of the oral cavity in a cohort of patients from Argentina with different oral and dental clinical manifestations. J Dent Sci Ther. 2016;1(1):18-25.

17. Maubon D, Garnaud C, Calandra T, Sanglard D, Cornet M. Resistance of Candida spp. to antifungal drugs in the ICU: where are we now? Intensive Care Med. 2014;40(9):1241-55.

18. Paul S, Kannan I. Molecular identification and antifungal susceptibility pattern of Candida species isolated from HIV infected patients with candisiasis. Curr Med Mycol. 2019;5(1):21-6.

19. Blot S, Vandijck D, Vandewoude K. Risk factors for Candida non-albicans candidemia. Diagn Microbiol Infect Dis. 2008;61(3):362-3.

20. Gong X, Luan T, Wu X, Li G, Qiu H, Kang Y, et al. Invasive candidiasis in intensive care units in China: risk factors and prognoses of Candida albicans and non-albicans Candida infections. Am J Infect Control. 2016;44(5):e59-63.
21. Muadcheingka T, Tantivitayakul P. Distribution of Candida albicans and non-albicans Candida species in oral candidiasis patients: correlation between cell surface hydrophobicity and biofilm forming activities. Arch Oral Biol. 2015;60(6):894-901.

22. Méan M, Marchetti O, Calandra T. Bench-to-bedside review: Candida infections in the intensive care unit. Crit Care. 2008;12(1):204.

23. Pfaller M, Diekema D. Epidemiology of invasive candidiasis: a persistent public health problem. Clin Microbiol Rev. 2007;20(1):133-63.

24. Singh T, Kashyap AK, Ahluwalia G, Chinna D, Sidhu SS. Epidemiology of fungal infections in critical care setting of a tertiary care teaching hospital in North India: a prospective surveillance study. Mortality. 2014. https:// doi.org/10.15380/2277-5706.jcsr.13.050.

25. Chakrabarti A, Chatterjee SS, Rao K, Zameer M, Shivaprakash M, Singhi S, et al. Recent experience with fungaemia: change in species distribution and azole resistance. Scand J Infect Dis. 2009;41(4):275-84.

26. Bhattacharjee P. Epidemiology and antifungal susceptibility of Candida species in a tertiary care hospital, Kolkata, India. Curr Med Mycol. 2016:2(2):20-7.

27. Kaur R, Dhakad MS, Goyal R, Kumar R. Emergence of non-albicans Candida species and antifungal resistance in intensive care unit patients. Asian Pac J Trop Biomed. 2016;6(5):455-60.

28. Al-Attas SA, Amro SO. Candidal colonization, strain diversity, and antifungal susceptibility among adult diabetic patients. Ann Saudi Med. 2010;30(2):101-8.

29. Pakshir K, Zomorodian K, Zakaei A, Motamedi M, Ghiasi MR, Karamitalab M. Molecular identification and in vitro antifungal susceptibility testing of Candida species isolated from patients with onychomycosis. Curr Med Mycol. 2015;1(4):26-32.

30. Apisarnthanarak A, Naknarongkij N, Kiratisin P, Mundy LM. Risk factors and outcomes of Candida albicans and non-albicans Candida species at a Thai tertiary care center. Am J Infect Control. 2009;37(9):781-2.

31. Serefhanoglu K, Timurkaynak F, Can F, Cagir U, Arslan H, Ozdemir FN. Risk factors for candidemia with non-albicans Candida spp. in intensive care unit patients with end-stage renal disease on chronic hemodialysis. J Formos Med Assoc. 2012;111(6):325-32.

32. Montagna M, Lovero G, Borghi E, Amato G, Andreoni S, Campion L, et al. Candidemia in intensive care unit: a nationwide prospective observational survey (GISIA-3 study) and review of the European literature from 2000 through 2013. Eur Rev Med Pharmacol Sci. 2014;18(5):661-74.

33. Tavanti A, Davidson AD, Gow NA, Maiden MC, Odds FC. Candida orthopsilosis and Candida metapsilosis spp. nov. to replace Candida parapsilosis groups II and III. J Clin Microbiol. 2005;43(1):284-92.

34. Mohammadi R, Mirhendi H, Rezaei-Matehkolaei A, Ghahri M, Shidfar MR, Jalalizand N, et al. Molecular identification and distribution profile of Candida species isolated from Iranian patients. Med Mycol. 2013;51(6):657-63.

35. Wolf DG, Falk R, Hacham M, Theelen B, Boekhout T, Scorzetti G, et al. Multidrug-resistant Trichosporon asahii infection of nongranulocytopenic patients in three intensive care units. J Clin Microbiol. 2001;39(12):4420-5.

36. Krcmery V Jr, Mateička F, Kunová A, Špánik S, Gyarfáš J, Syčová Z, et al. Hematogenous trichosporonosis in cancer patients: report of 12 cases including 5 during prophylaxis with itraconazol. Support Care Cancer. 1999;7(1):39-43.

37. Pfaller MA, Diekema DJ, Merz WG. Infections caused by non-Candida, non-Cryptococcus yeasts. 2nd ed. London: Churchill Livingstone; 2009.

\section{Publisher's Note}

Springer Nature remains neutral with regard to jurisdictional claims in published maps and institutional affiliations. 